\title{
METÁSTASIS HEPÁTICAS DE ORIGEN NO COLORRECTAL NI NEUROENDOCRINO. TRATAMIENTO EN UN HOSPITAL DE SEGUNDO NIVEL*
}

\author{
Drs. Antonio Palomeque J. ${ }^{1}$, Francisco Navarro F. ${ }^{1}$, Beatriz Pérez C. ${ }^{1}$, \\ Patricia Navarro S. ${ }^{2}$, Sra. Amanda Rocío González R. ${ }^{3}$, Dr. José Antonio Jiménez R. ${ }^{1}$ \\ Servicio de Cirugía General y Aparato Digestivo. Hospital Universitario San Cecilio. Granada. \\ 2 Servicio de Radiología. Hospital General Universitario Santa Lucía. Cartagena (Murcia). \\ ${ }^{3}$ Fundación Pública Andaluza para la Investigación Biosanitaria de Andalucía Oriental (FIBAO). \\ España.
}

\section{Abstract \\ Liver metastases from non-colorectal non-neuroendocrine tumors. Treatment in a hospital of second level}

Introduction: Surgical treatment of liver metastases is broadly accepted for colorectal and neuroendocrine tumors. However, the approach to liver metastases of other origins is not well established. The objective is the evaluation of factors that might be associated with better or worse perioperative outcomes (morbidity and mortality) and survival of patients with non-colorectal, non-neuroendocrine liver metastases (NCNNLM) treated surgically in a second level hospital. Material and Methods: A prospective study of patients with NCNNLM treated surgically or with radiofrequency was completed in San Cecilio University Hospital of Granada, between March 2003 and June 2013. The statistical analysis was made with IBM-SPSS version 21. Statistical significance was set at $\mathrm{p} \leq 0.05$. Survival data were analyzed by Kaplan-Meier's method. Results: Twenty-two patients with 26 surgeries were analyzed, 19 liver resections, 6 radiofrequency treatments and 1 combined case with surgery and radiofrequency. Operatory mortality was $0 \%$ and overage mortality was $23.1 \%$. Univariate analysis only showed statistical significance in two of the factors, presence of positive lymphatic nodes during the surgery of the primary tumor and presence of extra-hepatic disease. Accumulated survival was $81 \%, 66 \%$ and $52 \%$ at 1,2 and 3 years respectively. Conclusions: Despite the fact of treating together in the same group several different tumors, the surgical treatment of NCNNLM (with or without radiofrequency) has an acceptable survival rate. Thus, they can be treated with safety when we use a multidisciplinary approach to these patients.

Key words: Liver metastasis noncolorectal tumor hepatectomy, nonneuroendocrine tumor.

*Recibido el 8 de abril de 2014 y aceptado para publicación el 11 de junio de 2014.

Los autores declaran que no se han recibido apoyos financieros para la realización del estudio, ni existen conflictos de intereses.

Correspondencia: Dr. Antonio Palomeque J.

apalomeque2002@hotmail.com 


\section{Resumen}

Introducción: Aunque el tratamiento quirúrgico de las metástasis hepáticas está bien establecido en el cáncer colorrectal y los tumores neuroendocrinos, el enfoque de las metástasis hepáticas de otras localizaciones no está bien definido. El objetivo es valorar aquellos factores que pudieran asociarse a unos mejores o peores resultados perioperatorios (morbimortalidad) y a la supervivencia de los pacientes sometidos a tratamiento quirúrgico de las metástasis hepáticas de tumores no colorrectales ni neuroendocrinos(MHNCNN) en un hospital de segundo nivel. Material y Métodos: Se ha realizado un estudio prospectivo de pacientes con MHNCNN tratadas mediante cirugía y/o radiofrecuencia en el Hospital Universitario San Cecilio de Granada, entre marzo de 2003 y junio de 2013. Para el análisis estadístico se utilizó el programa IBM-SPSS versión 21 , considerándose significativo un valor de $\mathrm{p} \leq 0,05$. El estudio de supervivencia se realizó mediante análisis de Kaplan-Meier. Resultados: Se analizan 22 pacientes, con 26 intervenciones: 19 resecciones quirúrgicas, 6 radiofrecuencias y 1 caso combinando resección y radiofrecuencia. La mortalidad operatoria es de $0 \%$ y la morbilidad del 23\%. El análisis univariante sólo muestra significación estadística en dos factores, presencia de ganglios positivos en la intervención del tumor primario y la existencia de enfermedad extrahepática. La supervivencia acumulada es del 81\%, 66\% y 52\% al año, dos años y tres años. Conclusiones: Aunque con las limitaciones que presenta el agrupar un conjunto heterogéneo de tumores, el tratamiento quirúrgico de las MHNCNN, asociado o no a radiofrecuencia, presenta una tasa de supervivencia aceptable, pudiendo ser estas tratadas con seguridad, siendo fundamental un abordaje multidisciplinario de los pacientes.

Palabras clave: Metástasis hepáticas, tumor no colorrectal, tumor no neuroendocrino, hepatectomía.

\section{Introducción}

El hígado es una localización frecuente de las metástasis de tumores de diferentes orígenes, aunque los mecanismos para el desarrollo de las metástasis hepáticas $(\mathrm{MH})$ difieren dependiendo de la localización del tumor primario. En pacientes con tumores primarios del tracto gastrointestinal, como el adenocarcinoma colorrectal y los tumores neuroendocrinos asociados al intestino, el modo más probable de propagación al hígado es a través de drenaje venoso portal o a través de canales linfáticos intraabdominales directos. En estos casos, la resección hepática de las metástasis se basa en que la mayor parte de la carga tumoral en el paciente está limitada al abdomen, por lo que un tratamiento adecuado del tumor primario, en combinación con la resección de las $\mathrm{MH}$, puede plantearse con posibilidades de curación. Por otro lado, un elevado número de $\mathrm{MH}$ proceden de tumores originados fuera de la cavidad abdominal que, por lo general, utilizan la circulación sistémica para alcanzar el hígado, pudiéndose afectar otras localizaciones extrahepáticas con igual probabilidad ${ }^{1-3}$.

Los primeros trabajos sobre $\mathrm{MH}$ no colorrectales tratados mediante resección hepática incluyeron tumores neuroendocrinos y no neuroendocrinos, mostrando mejor pronóstico el grupo de $\mathrm{MH}$ de origen neuroendocrino ${ }^{3-8}$. Estudios posteriores han confirmado estas diferencias de supervivencia y han excluido a los pacientes con $\mathrm{MH}$ de origen neuroendocrino, mostrando que la hepatectomía por $\mathrm{MH}$ no colorrectales no neuroendocrinas (MHNCNN) es factible y segura, siendo un tratamiento que puede aumentar la supervivencia, consiguiéndose cifras a 5 años entre el 19 y el $45 \%{ }^{9-23}$ (Tabla 1 ). Se estima que sólo el $10-15 \%$ de los pacientes son candidatos a resecciones completas (R0) ${ }^{19}$, aunque con el desarrollo de la embolización portal, la asociación de resección quirúrgica más ablación por radiofrecuencia $(\mathrm{RF})$ y el uso de la ecografía intraoperatoria, han aumentado las posibilidades de resección, en particular de los pacientes con $\mathrm{MH}$ múltiples bilaterales ${ }^{24}$. La mayoría de los trabajos publicados se limitan, esencialmente, a los estudios de un único centro, existiendo escasos trabajos multicéntricos con un elevado número de pacientes, por lo que se necesita una mejor comprensión de los beneficios potenciales de la resección quirúrgica para estos tumores.

Nuestro objetivo es valorar aquellos factores que pudieran asociarse a unos mejores o peores resultados perioperatorios (morbimortalidad) y a la supervivencia de los pacientes sometidos a tratamiento quirúrgico, acompañado o no de RF, de las MHNCNN en un hospital de segundo nivel.

\section{Material y Métodos}

\section{Pacientes}

Se realiza un estudio retrospectivo de los pacientes con MHNCNN tratados mediante cirugía y/o RF en el Hospital Universitario San Cecilio de Granada (España), centro hospitalario de segundo nivel, desde marzo de 2003 a junio de 2013. La información fue recogida en una base de datos previamente diseñada, conteniendo datos demográficos, estirpe, estadío y características del tumor primario, 
Tabla 1. Trabajos científicos publicados sobre resección quirúrgica de metástasis hepáticas de origen no colorrectal ni neuroendocrino

\begin{tabular}{|c|c|c|c|c|c|}
\hline Primer autor & Año & $\mathbf{n}$ & $\begin{array}{c}\text { Morbilidad } \\
\text { operatoria (\%) }\end{array}$ & $\begin{array}{c}\text { Mortalidad } \\
\text { operatoria (\%) }\end{array}$ & $\begin{array}{c}\text { Supervivencia } \\
\text { a los } 5 \text { años }(\%)\end{array}$ \\
\hline Harrison ${ }^{9}$ & 1997 & 96 & NI & 0 & 37 \\
\hline Hemming $^{10}$ & 2000 & 37 & NI & 0 & 45 \\
\hline Yamada $^{11}$ & 2001 & 33 & 21 & 9 & 12 \\
\hline Laurent $^{12}$ & 2001 & 39 & 8 & 0 & 35 \\
\hline Takada $^{13}$ & 2001 & 14 & NI & 7 & NI \\
\hline Karavias $^{14}$ & 2002 & 18 & 11 & 0 & NI \\
\hline Ercolani $^{15}$ & 2005 & 142 & 21 & 0 & 34 \\
\hline Cordera $^{16}$ & 2005 & 64 & 7 & 2 & 30 \\
\hline Weitz ${ }^{17}$ & 2005 & 141 & 33 & 0 & NI \\
\hline $\mathrm{Teo}^{18}$ & 2006 & 18 & 0 & 0 & NI \\
\hline Adam $^{19}$ & 2006 & 1.452 & 22 & 2 & 36 \\
\hline Lendoire $^{20}$ & 2007 & 106 & NI & 2 & 19 \\
\hline $\mathrm{O}^{\prime}$ Rourke $^{21}$ & 2008 & 102 & 21 & 1 & 39 \\
\hline Ercolani $^{22}$ & 2009 & 134 & 23 & 3 & 40 \\
\hline Yáñez ${ }^{23}$ & 2010 & 17 & 6 & 0 & $50,9 *$ \\
\hline Groeschl $^{3}$ & 2012 & 420 & 20 & 2 & 31 \\
\hline
\end{tabular}

NI: No indicado en el trabajo. *Supervivencia a los 3 años.

marcadores tumorales, tiempo libre de enfermedad tras intervención del tumor primario, métodos diagnósticos, tiempo entre el diagnóstico de las $\mathrm{MH}$ y la intervención quirúrgica, intervención realizada, requerimiento transfusional, márgenes quirúrgicos, morbimortalidad, estancia hospitalaria y estado actual de los pacientes.

Todos los pacientes han sido evaluados por un equipo multidisciplinario y han recibido tratamiento adyuvante según el tipo de tumor primario.

Se definió metástasis hepática sincrónica la diagnosticada en los primeros 6 meses tras la cirugía del tumor primario y metacrónica, aquella que se diagnosticó después de los 6 meses.

Las complicaciones postoperatorias fueron descritas siguiendo los criterios de Clavien ${ }^{25}$.

Son consideradas MH resecables las que se prevé una resección completa de las mismas, basada en estudios por imagen preoperatorios, garantizándose una vascularización, drenaje biliar y tejido hepático remanente adecuado (mayor del 20\% del volumen hepático estimado y mayor del $40 \%$ en pacientes con hepatopatía crónica).

Fueron excluidos los pacientes con invasión directa del tejido hepático por un tumor de origen extrahepático, aquellos que presentaban enfermedad extrahepática diseminada sin posibilidad de resección quirúrgica y los que, durante el tratamiento adyuvante (o neoadyuvante para la $\mathrm{MH}$ ), presentaron progresión de la enfermedad.

La evaluación del paciente se realizó en todos los casos con ecografía y tomografía axial computarizada (TC) abdominopélvica y de tórax, completándose con resonancia nuclear magnética (RNM) y tomografía por emisión de positrones (PET) en determinados pacientes. Se realizó ecografía intraoperatoria en todos los pacientes.

\section{Análisis estadístico}

Para el análisis estadístico se utilizó el programa IBM-SPSS versión 21, aplicándose pruebas estadísticas no paramétricas (Mann-Whitney y KruskalWallis) por no tener un tamaño muestral superior a 30 pacientes en cada uno de los grupos. Se ha considerado como significativo un valor de $p \leq 0,05$. El estudio de supervivencia se realizó mediante un análisis de Kaplan-Meier. Las variables cuantitativas son expresadas mediante la media y la mediana, como medidas de tendencia central, y con la desviación típica y el rango como medidas de dispersión, mientras que las variables cualitativas se expresan mediante frecuencias. 


\section{Resultados}

\section{Pacientes y características del tumor}

Se analizaron los datos de los pacientes con MH intervenidos entre marzo de 2003 y junio de 2013. Durante este período se realizaron 211 intervenciones quirúrgicas por $\mathrm{MH}$, de las que 26 fueron por MHNCNN (12,3\%). Estas 26 intervenciones de MHNCNN se realizaron sobre 22 pacientes, sometiéndose 19 pacientes a una única intervención por $\mathrm{MH}$ y 3 , a rehepatectomía ( 2 pacientes a una rehepatectomía y 1 paciente a dos). La edad media fue de 53,1 \pm 9 años. Los resultados del estudio descriptivo de los pacientes y características del tumor se recogen en la Tabla 2 y la localización del tumor primario, en la Tabla 3 . Todos los pacientes fueron valorados por un equipo multidisciplinar, recibido el tratamiento neoadyuvante y adyuvante correspondiente a la histología de cada tipo de tumor primario.

El tiempo libre de enfermedad presenta una mediana de 37,2 meses (rango: 10-83), excluyendo los pacientes que presentaban las $\mathrm{MH}$ cuando se realizó el diagnóstico del tumor primario y que se sometieron en el mismo acto quirúrgico a la resección de las $\mathrm{MH}$. En relación con los datos que aparecen en la Tabla 2, indicar que los 7 pacientes incluidos en el grupo de MH sincrónicas, todos los casos corresponden con $\mathrm{MH}$ que ya aparecen en la intervención del tumor primario, no apareciendo casos de $\mathrm{MH}$ en los primeros 6 meses.

La mediana del tamaño de las metástasis es de $23 \mathrm{~mm}$ (rango: 6-100).

\section{Tratamiento quirúrgico/resección hepática}

Para la resección hepática se utilizó la clasificación anatómica de Couinaud ${ }^{26}$, así como
Tabla 2. Estudio descriptivo de variables cualitativas de los pacientes, características del tumor primario y metástasis hepáticas

\begin{tabular}{|c|c|c|c|}
\hline Variable & & n* & $\%$ \\
\hline Sexo & Hombre & 10 & 45,5 \\
\hline & Mujer & 12 & 54,5 \\
\hline Clasificación ASA & I & 1 & 4,5 \\
\hline & II & 10 & 45,5 \\
\hline & III & 11 & 50 \\
\hline Histología tumor primario & $\begin{array}{l}\text { Adenocarcino- } \\
\text { ma }\end{array}$ & 15 & 68,2 \\
\hline & Otros & 7 & 31,8 \\
\hline Estadío tumor primario & I-II & 6 & 27,3 \\
\hline & III-IV & 16 & 72,7 \\
\hline Invasión linfática tumor & Sí & 16 & 72,7 \\
\hline primarıo & No & 6 & 27,3 \\
\hline Invasión vascular tumor & Sí & 17 & 77,3 \\
\hline primario & No & 5 & 22,7 \\
\hline Tipo de metástasis & Sincrónica & 7 & 68,2 \\
\hline & Metacrónica & 15 & 31,8 \\
\hline Número de metástasis & Única & 9 & 34,6 \\
\hline & Múltiple & 17 & 65,4 \\
\hline Extensión afectación & Unilobar & 20 & 76,9 \\
\hline hepatıca & Bilobal & 6 & 23,1 \\
\hline Enfermedad extrahepática & Sí & 8 & 36,4 \\
\hline intervencion tumor primario & No & 14 & 63,6 \\
\hline
\end{tabular}

*n es 22 ó 26 según se trate de pacientes o procedimientos realizados, respectivamente.

Tabla 3. Distribución de los pacientes según localización del tumor primario

\begin{tabular}{|llcc|}
\hline Localización tumor primario & Órgano tumor primario & n de pacientes & $\%$ \\
Extradigestivo & Tiroides & 1 & 4,5 \\
& Glándula suprarrenal & 1 & 4,5 \\
& Mama & 3 & 13,5 \\
& Melanoma & 2 & 9 \\
& Ovario & 3 & 13,5 \\
Digestivo & Pulmón & 1 & 4,5 \\
& Estómago & 5 & 22,5 \\
& Vesícula & 1 & 4,5 \\
& Esófago & 2 & 9 \\
& Desconocido (páncreas) & 1 & 4,5 \\
& Páncreas & 1 & 4,5 \\
& Colangiocarcinoma & 1 & 4,5 \\
\hline
\end{tabular}


la clasificación de resecciones hepáticas del Consenso de Brisbane ${ }^{27}$. De las 26 intervenciones por MHNCNN, 24 se realizaron mediante laparotomía y 2 por un abordaje laparoscópico, consistentes en 2 ablaciones por radiofrecuencia. El tiempo transcurrido entre el diagnóstico de las metástasis y la intervención quirúrgica presentó una mediana de 3,6 meses (rango: 1-9), para aquellos casos en los que la intervención del tumor primario y las $\mathrm{MH}$ no se realizó en una misma intervención quirúrgica. Del total de 26 intervenciones realizadas por MHNCNN se realizaron 6 intervenciones en las que el tratamiento único fue la RF, con una mediana de 2 metástasis hepáticas tratadas (rango: 1-7). En 1 intervención se realizó tratamiento combinado con RF $(2 \mathrm{MH})$ y resección hepática (2 resecciones atípicas). En las 19 intervenciones restantes, el tratamiento se basó en la resección hepática, con una mediana de $2 \mathrm{MH}$ tratadas (rango 1-7), realizándose una resección mayor en 5 intervenciones (5 lobectomías izquierdas) y una resección menor en 14 de las intervenciones.

En 17 intervenciones quirúrgicas no fue necesario realizar transfusión sanguínea y, en 9 casos, sí fue necesaria dicha transfusión. La mediana de transfusiones fue de 2 concentrados de hematíes (rango: 2-5).

De los 20 pacientes resecados, en 19 casos los márgenes de resección fueron superiores a $10 \mathrm{~mm}$ (resección R0) y, sólo en 1 de los casos, el borde de resección contactaba con la lesión metastásica (resección R1).

Durante los dos meses tras la cirugía, la mortalidad fue del $0 \%$. La morbilidad fue del 23\%, 5 complicaciones grado II y 1 , grado IIIa, resolviéndose todas de forma médica.

La estancia total de las 26 intervenciones presenta una mediana de $7\left(\mathrm{P}_{25}=5 ; \mathrm{P}_{75}=10\right)$. Excluyendo los casos de cirugía simultánea del tumor primario y las $\mathrm{MH}$, la estancia media presenta una mediana de 6 $\left(\mathrm{P}_{25}=3 ; \mathrm{P}_{75}=8\right)$.

\section{Supervivencia}

Tras un seguimiento medio de 34 meses, la mediana de la supervivencia es de 23,2 meses (rango: 3-99). La mayor supervivencia de la serie corresponde a un paciente con un adenocarcinoma gástrico. Al concluir el estudio, 11 pacientes permanecían vivos (50\%). El análisis de supervivencia mediante Kaplan-Meier muestra una supervivencia acumulada del $81 \%$ al año de seguimiento, disminuyendo al $66 \%$ a los dos años y al 52\% a los 5 años (Figura 1). Tres de los 11 pacientes vivos se encuentran libres de enfermedad, con un seguimiento medio de 43 meses y 8 presentan progresión de la enfermedad ( 5 con MH múltiples y 3 pacientes, dos con carcinoma de ovario y una con carcinoma suprarrenal, con MH y enfermedad extrahepática). Tres pacientes se sometieron a rehepatectomía (dos de ellos a una y otro paciente a dos), falleciendo los dos primeros; la tercera, correspondiente a la enferma con el adenocarcinoma de la corteza suprarrenal, se encuentra viva, con enfermedad hepática y retroperitoneal.

Para determinar los posibles factores que podrían influir en la supervivencia de los pacientes tratados quirúrgicamente $\mathrm{y} / \mathrm{o}$ mediante $\mathrm{RF}$, se ha realizado un análisis bivariante, mostrando solamente una correlación estadísticamente significativa entre la supervivencia y la presencia de ganglios positivos en la intervención del tumor primario y entre la supervivencia y la presencia de enfermedad extrahepática en la cirugía del tumor primario. En ambos casos, la supervivencia es inferior cuando existen ganglios

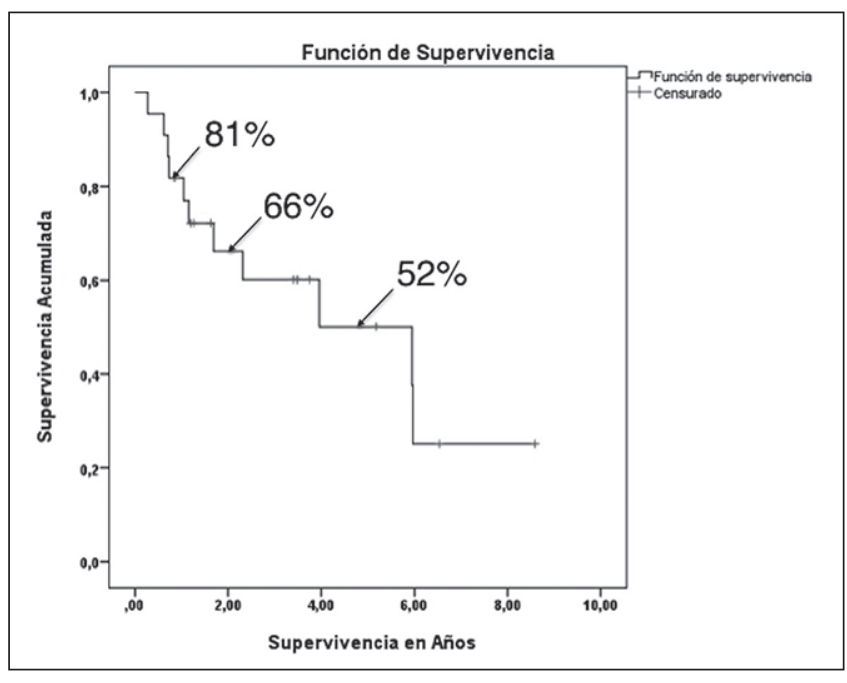

Figura 1. Supervivencia acumulada en pacientes sometidos a tratamiento quirúrgico por metástasis hepáticas de origen no colorrectal ni neuroendocrino. 
Tabla 4. Análisis bivariante de los factores determinantes en la sobrevida en pacientes con MHNCNN sometidos a tratamiento quirúrgico/RF

\begin{tabular}{|c|c|c|c|c|c|}
\hline Categoría & Factor pronóstico & Estrato & n & Test estadístico & Valor $\mathbf{p}$ \\
\hline \multirow[t]{3}{*}{ Paciente } & Edad & $\begin{array}{l}<45 \mathrm{a} \\
>45 \mathrm{a}\end{array}$ & $\begin{array}{c}6 \\
16\end{array}$ & U de Mann-Whitney & 0,367 \\
\hline & Sexo & $\begin{array}{l}\text { Hombre } \\
\text { Mujer }\end{array}$ & $\begin{array}{l}10 \\
12\end{array}$ & U de Mann-Whitney & 0,798 \\
\hline & ASA & $\begin{array}{l}\text { I-II } \\
\text { III-IV }\end{array}$ & $\begin{array}{l}11 \\
11\end{array}$ & U de Mann-Whitney & 0,898 \\
\hline \multirow[t]{5}{*}{$\begin{array}{l}\text { Tumor } \\
\text { primario }\end{array}$} & Localización & $\begin{array}{l}\text { Digestivo } \\
\text { Extradigestivo }\end{array}$ & $\begin{array}{l}11 \\
11\end{array}$ & U de Mann-Whitney & 0,847 \\
\hline & Tipo histológico & $\begin{array}{l}\text { Adenocarcinoma } \\
\text { Otro tipo }\end{array}$ & $\begin{array}{c}15 \\
7\end{array}$ & U de Mann-Whitney & 0,783 \\
\hline & Estadío & $\begin{array}{l}\text { I-II } \\
\text { III-IV }\end{array}$ & $\begin{array}{c}6 \\
16\end{array}$ & U de Mann-Whitney & 0,693 \\
\hline & Ganglios & $\begin{array}{l}\text { Positivos } \\
\text { Negativos }\end{array}$ & $\begin{array}{c}16 \\
6\end{array}$ & U de Mann-Whitney & 0,049 \\
\hline & Enfermedad extrahepática & $\begin{array}{l}\text { Sí } \\
\text { No }\end{array}$ & $\begin{array}{c}8 \\
14\end{array}$ & U de Mann-Whitney & 0,025 \\
\hline \multirow[t]{5}{*}{$\begin{array}{l}\text { Metástasis } \\
\text { hepáticas }\end{array}$} & Intervalo libre enfermedad & $\begin{array}{l}\leq 12 \text { meses } \\
12-24 \text { meses } \\
\geq 24 \text { meses }\end{array}$ & $\begin{array}{c}14 \\
1 \\
7\end{array}$ & Kruskal-Wallis & 0,363 \\
\hline & Número metástasis $1^{\mathrm{a}}$ intervención & $\begin{array}{l}\text { Única } \\
\text { Múltiple }\end{array}$ & $\begin{array}{c}7 \\
15\end{array}$ & U de Mann-Whitney & 0,490 \\
\hline & Tipo & $\begin{array}{l}\text { Metacrónica } \\
\text { Sincrónica }\end{array}$ & $\begin{array}{c}15 \\
7\end{array}$ & U de Mann-Whitney & 0,185 \\
\hline & Tamaño metástasis mayor $1^{\mathrm{a}}$ intervención & $\begin{array}{l}<50 \mathrm{~mm} \\
>50 \mathrm{~mm}\end{array}$ & $\begin{array}{c}19 \\
3\end{array}$ & U de Mann-Whitney & 0,701 \\
\hline & Lateralidad $1^{\mathrm{a}}$ intervención & $\begin{array}{l}\text { Unilobales } \\
\text { Bilobales }\end{array}$ & $\begin{array}{c}18 \\
4\end{array}$ & U de Mann-Whitney & 0,971 \\
\hline \multirow[t]{4}{*}{$\begin{array}{l}\text { Tratamiento } \\
\text { metástasis }\end{array}$} & Cirugía y/o RF $1^{a}$ intervención & $\begin{array}{l}\text { Cirugía } \\
\text { RF } \\
\text { Cirugía+RF }\end{array}$ & $\begin{array}{c}15 \\
6 \\
1\end{array}$ & Kruskal-Wallis & 0,401 \\
\hline & Extensión hepatectomía 1a intervención* & $\begin{array}{l}\text { Mayor } \\
\text { Limitada }\end{array}$ & $\begin{array}{c}5 \\
15\end{array}$ & U de Mann-Whitney & 1,000 \\
\hline & Márgenes resección quirúrgica $1^{\mathrm{a}}$ intervención* & $\begin{array}{l}\text { R0 } \\
\text { R1 }\end{array}$ & $\begin{array}{c}19 \\
1\end{array}$ & U de Mann-Whitney & 0,596 \\
\hline & Rehepatectomía & $\begin{array}{l}\text { Sí } \\
\text { No }\end{array}$ & $\begin{array}{c}3 \\
19\end{array}$ & U de Mann-Whitney & 0,191 \\
\hline
\end{tabular}

$*_{\mathrm{n}}=20$, referido a casos en los que sólo se realizó algún tratamiento quirúrgico de las MH y no RF.

positivos y cuando existe enfermedad extrahepática. (Tabla 4).

\section{Discusión}

La resección hepática por $\mathrm{MH}$ colorrectales se ha establecido como una práctica segura, con re- sultados favorables en pacientes cuidadosamente seleccionados $^{28-30}$, al igual que ocurre con las $\mathrm{MH}$ de tumores neuroendocrinos ${ }^{4-8}$. En el caso de los tumores primarios extraabdominales, por la posible diseminación a regiones extrahepáticas de forma inicial ${ }^{1,2}$, se han planteado, históricamente, dudas sobre la utilidad del tratamiento quirúrgico de las $\mathrm{MH}^{31}$. Actualmente, debido a la falta de datos que 
apoyen otras modalidades terapéuticas, la mejora de los estudios preoperatorios y la seguridad de las técnicas de la cirugía hepática, el tratamiento de las MHNCNN está cobrando entusiasmo.

El presente trabajo recoge la experiencia de un centro hospitalario de segundo nivel en el que se ha realizado el tratamiento quirúrgico de las MHNCNN de 22 pacientes, con un total de 26 intervenciones, incluyendo resecciones hepáticas, acompañadas o no por RF y 6 casos en los que el tratamiento exclusivo con RF. Con el desarrollo de la embolización portal, la asociación de resección quirúrgica más RF y el uso de la ecografía intraoperatoria, han aumentado las posibilidades de resección, en particular de los pacientes con metástasis múltiples bilaterales ${ }^{24}$, utilidad que ya estaba reconocida para la RF en tumores hepáticos primarios y metastásicos de origen colorrectal ${ }^{32}$. Sin existir muchos estudios sobre el uso de la RF para el tratamiento de MHNCNN, encontramos en la literatura algunos sobre las $\mathrm{MH}$ del cáncer gástrico, mama y colangiocarcinoma, con buenos resultados ${ }^{33,34}$. En nuestra serie la RF se utilizó como complementario a la cirugía en un caso y, en 6 casos, como único tratamiento, pacientes estos últimos que no se consideraron aptos para poder realizar una resección quirúrgica, por ser MH bilobales o de localización que imposibilitaba la cirugía.

La supervivencia acumulada ha sido del $81 \%$ al año, $66 \%$ a los 2 años y $52 \%$ a los 5 años, con tres pacientes que sobreviven libres de enfermedad al final de nuestro estudio, con un seguimiento de 15, 41 y 75 meses y que se corresponden con pacientes intervenidos por MH de cáncer de vesícula, gástrico y mama respectivamente, poniendo de manifiesto que la resección de las MHNCNN proporciona sobrevidas a tener en cuenta. En la Tabla 1 se muestran otros estudios, observándose cómo en las series de mayor número de pacientes la supervivencia a los 5 años máxima es del $45 \%$, estando por debajo de lo mostrado en nuestra serie (52\%), aunque esta comparación en pacientes con MHNCNN puede estar interferido por la diversidad de tipos de tumores incluidos en cada serie, con diferente comportamiento biológico.

En relación a la seguridad del tratamiento quirúrgico, en nuestra serie no se presenta ningún caso de mortalidad operatoria y la morbilidad es del $23 \%$, datos similares a los reportados en otros trabajos, en los que la mortalidad muestra valores de $0-9 \%$ y la morbilidad de $0-33 \% 0^{3,9-23}$.

En nuestra serie, únicamente dos factores han presentado significación en relación a una supervivencia inferior, la presencia de ganglios positivos en la cirugía del tumor primario y la presencia de enfermedad extrahepática en la cirugía de las $\mathrm{MH}$, en concordancia con otros trabajos ${ }^{19-22}$.
De este modo, nuestros resultados, aún encontrándose en la misma línea de otros trabajos publicados, requieren ciertas reflexiones. En varias publicaciones ${ }^{19,22}$, se sugiere un peor pronóstico cuando los tumores primarios son de origen gástrico o esofágico, que en nuestro estudio se cumple, con la excepción de un paciente con cáncer gástrico que presenta una supervivencia superior a 4 años, falleciendo por la diseminación hepática. Se han incluido dos pacientes con carcinoma pancreático, que aunque a priori no estaría indicada la cirugía al presentar $\mathrm{MH}$, se incluyeron en el tratamiento quirúrgico excepcionalmente, tratándose de un paciente de 30 años, con una MH única que se resecó y, un segundo caso, en que el diagnóstico de carcinoma de páncreas se obtuvo tras la cirugía de una $\mathrm{MH}$ sin origen conocido.

Algunos estudios ${ }^{19-21}$ muestran como factor de mejor supervivencia la existencia de un intervalo libre de enfermedad prolongado, en nuestra serie no ocurre lo mismo. Igualmente, no se han visto diferencias significativas en relación a los bordes de la resección hepática, al tipo de tumor primario y la histología del mismo en nuestro trabajo, factores que sí aparecen determinantes en otros estudios ${ }^{19,22}$.

Los pacientes con recurrencia de la enfermedad hepática suele ser indicativo de peor pronóstico, aunque son varios los autores que han señalado que la hepatectomía de repetición puede mejorar la supervivencia ${ }^{19}$. Nuestros pacientes sometidos a rehepatectomía presentaron una sobrevida elevada $\mathrm{y}$ uno de los pacientes permanece vivo al finalizar el estudio, aunque presenta recidiva tumoral hepática y retroperitoneal, correspondiendo a una paciente con un adenocarcinoma de la corteza suprarrenal, mejor supervivencia que también exponen algunas publicaciones $^{35}$.

Como limitaciones de nuestro trabajo estarían el reducido número de casos tratados de MHNCNN en nuestro centro hospitalario, la elevada variedad histológica de tumores, con diferente comportamiento biológico, y el largo período recogido, 10 años, en los que el arsenal terapéutico oncológico para estos tipos de tumores ha ido variando de forma significativa. Igualmente se han incluido pacientes sometidos a tratamiento quirúrgico y pacientes sometidos a RF, con escasos pacientes en cada grupo, por los que los resultados en la comparación de ambos también se ve limitada, necesitándose ampliar nuestra serie de pacientes para poder obtener unos resultados más concluyentes, si bien estos son alentadores.

Con todo lo expuesto, podríamos decir que el antiguo dogma de que "No hay papel en el tratamiento quirúrgico de las metástasis hepáticas de los tumores no colorrectales no neuroendocrinos" ha dejado de ser válido en la actualidad, ya que la cirugía de las 
metástasis en estos pacientes está aportando sobrevidas importantes. A diferencia de lo que ocurre en el cáncer de colon, donde la cirugía de las metástasis juega un papel clave y la quimioterapia actúa como adyuvante, en los tumores no neuroendocrinos no colorrectales, podríamos decir que el tratamiento sistémico es la base del tratamiento y, una vez estabilizada la enfermedad, la cirugía actúa complementando a ésta ${ }^{19}$. El tratamiento de las MHNCNN debe llevarse a cabo en aquellos centros en los que exista un equipo multidisciplinario que valore al paciente de manera individual y equipos quirúrgicos expertos en cirugía hepática en los que se cumplan los estándares de calidad ya establecidos para la cirugía de las metástasis hepáticas de origen colorrectal.

\section{Conclusiones}

Las MHNCNN representan un estadío avanzado de numerosos tumores malignos, pudiéndose tratar con seguridad y con unas tasas de supervivencia aceptables. El abordaje multidisciplinario de todos los pacientes es fundamental para un tratamiento óptimo de estos pacientes, en los que la hepatectomía y la ablación por radiofrecuencia en casos seleccionados debe ser considerada, junto con un tratamiento oncológico adecuado.

\section{Referencias}

1. Belghiti J, Hiramatsu K, Benoist S, Massault P, Sauvanet A, Farges O. Seven hundred forty-seven hepatectomies in the 1990s: an update to evaluate the actual risk of liver resection. J Am Coll Surg. 2000;191:38-46.

2. Jarnagin WR, Gonen M, Fong Y, DeMatteo RP, BenPorat L, Little S, et al. Improvement in perioperative outcome after hepatic resection: analysis of 1803 consecutive cases over the past decade. Ann Surg. 2002;236:397-406.

3. Groeschl RT, Nachmany I, Steel JL, Reddy SK, Glazer ES, de Jong MC, et al. Hepatectomy for non-colorectal non-neuroendocrine metastatic cancer: a multi-institutional analysis. J Am Coll Surg. 2013;214:769-77.

4. Paineau J, Hamy A, Savigny B, Visset J. Resection of hepatic metastases from non colorectal cancers: our experience apropos of 20 cases. J Chir. 1995;132:1-6.

5. Berney T, Mentha G, Roth AD, Morel P. Results of surgical resection of liver metastases from non-colorectal primaries. Br J Surg. 1998;85:1423-7.

6. Lindell G, Ohlsson B, Saarela A, Andersson R, Tranberg KG. Liver resection of noncolorectal secondaries. J Surg Oncol. 1998;69:66-70.

7. Hamy AP, Paineau JR, Mirallie EC, Bizouarn P, Visset JP. Hepatic resections for non-colorectal metastases: forty resections in 35 patients. Hepatogastroenterology 2000;47:1090-4.

8. Goering JD, Mahvi DM, Niederhuber JE, Chicks D, Rikkers LF. Cryoablation and liver resection for noncolorectal liver metastases. Am J Surg. 2002;183:3849.

9. Harrison LE, Brennan MF, Newman E, Fortner JG, Picardo A, Blumgart LH, et al. Hepatic resection for noncolorectal, nonneuroendocrine metastases: a fifteen-year experience with ninety-six patients. Surgery 1997;121:625-32.

10. Hemming AW, Sielaff TD, Gallinger S, Cattral MS, Taylor BR, Greig PD, et al. Hepatic resection of noncolorectal nonneuroendocrine metastases. Liver Transpl. 2000;6:97-101.

11. Yamada H, Katoh H, Kondo S, Okushiba S, Morikawa T. Hepatectomy for metastases from non-colorectal and non-neuroendocrine tumors. Anticancer Res. 2001;21:4159-62.

12. Laurent C, Rullier E, Feyler A, Masson B, Saric J. Resection of noncolorectal and nonneuroendocrine liver metastases: late metastases are the only chance of cure. World J Surg. 2001;25:1532-6.

13. Takada Y, Otsuka M, Seino K, Taniguchi H, Koike N, Kawamoto T, et al. Hepatic resection for metastatic tumors from noncolorectal carcinoma. Hepatoenterology 2001;48:83-6.

14. Karavias DD, Tepetes K, Karatzas T, Felekouras E, Androulakis J. Liver resection for metastatic non-colorectal non-neuroendocrine hepatic neoplasms. Eur J Sur Oncol. 2002;28:135-9.

15. Ercolani G, Grazi GL, Ravaioli M, Ramacciato G, Cescon M, Varotti G, et al. The role of liver resections for noncolorectal, nonneuroendocrine metastases: experience with 142 observed cases. Ann Surg Oncol. 2005;12:459-66.

16. Cordera F, Rea DJ, Rodríguez-Dávalos M, Hoskin TL, Nagorney DM, Que FG. Hepatic resection for noncolorectal, nonneuroendocrine metastases. J Gastrointest Surg. 2005;9:1361-70.

17. Weitz J, Blumgart LH, Fong Y, Jarnagin WR, D'Angelica M, Harrison LE, et al. Partial hepatectomy for metastases from noncolorectal, nonneuroendocrine carcinoma. Ann Surg. 2005;241:269-76.

18. Teo MC, Tan YM, Chung AY, Chow PK, Cheow $\mathrm{PC}$, Soo KC, et al. Metastectomy for non-colorectal, non-neuroendocrine liver secondaries. ANZ J Surg. 2006;76:575-8.

19. Adam R, Chiche L, Aloia T, Elias D, Salmon R, Rivoire $\mathrm{M}$, et al. Hepatic resection for noncolorectal nonendocrine liver metastases: analysis of 1,452 patients and development of a prognostic model. Ann Surg. 2006;244:524-35.

20. Lendoire J, Moro M, Andriani O, Grondona J, Gil O, Raffin G, et al. Liver resection for non-colorectal, nonneuroendocrine metastases: analysis of a multicenter 
study from Argentina. HPB 2007;9:435-9.

21. O'Rourke TR, Tekkis P, Yeung S, Fawcett J, Lynch S, Strong R, et al. Long-term results of liver resection for non-colorectal, non-neuroendocrine metastases. Ann Surg Oncol. 2008;15:207-18.

22. Ercolani G, Vetrone G, Grazi GL, Cescon M, Di Giola $\mathrm{P}$, Ravioli $\mathrm{M}$, et al. The role of liver surgery in the treatment of non-colorectal non-neuroendocrine metastases (NCRNNE). Analysis of 134 resected patients. Minerva Chir. 2009;64:551-8.

23. Yáñez R, Gamboa C, Crovari F, Guzmán S, Martínez J, Jarufe N, y cols. Metastasis hepática de origen no colorrectal ni neuroendocrino. Tratamiento quirúrgico. Rev Chil Cir. 2002;62:27-32.

24. Fuente M. Manejo de las metástasis hepáticas de origen no colorrectales y no neuroendocrinas. Cirujano General 2011;33:20-2.

25. Dindo D, Demartines N, Clavien PA. Classification of surgical complications: a new proposal with evaluation ni a cohort of 6.336 patients and results of a survey. Ann Surg. 2004;240:5.

26. Couinaud C. The anatomy of the liver. Ann Ital Chir. 1992;63:693-7.

27. Belghiti J, Clavien PA, Gadzijer E, Garden JO, Lau WY, Makuuchi M. The Brisbane 2000 terminology of liver anatomy and resections. HPB. 2000;2:333-9.

28. Zhou L, Zhag G, Tang T, Lian R, Wang W. Retrospective analysis of clinical and pathologic risk factors in liver resection for hepatic colorectal metastases. J Cancer Res Ther. 2013;9:178-82.
29. De Haas RJ, Wicherts DA, Andreani P, Pascal G, Saliba $F$, Ichai $P$, et al. Impact of expanding criteria for resectability of colorectal metastases on short- and long-term outcomes after hepatic resection. Ann Surg. 2011;253:1069-79.

30. Wicherts DA, de Haas RJ, Salloum C, Andreani P, Pascal G, Sotirov D, et al. Repeat hepatectomy for recurrent colorectal metastases. Br J Surg. 2013;100:808-18.

31. Durán H, Bellón J. Papel de la cirurgía en las metástasis del cáncer de mama. Cir Esp. 2007;82:3-10.

32. Earle SA, Pérez EZ, Gutiérrez JC. Hepatectomy enables prolonged a survival in select patients with isolated non-colorectal liver metastasis. Am Coll Surg. 2006;203:436-46.

33. Rose DM, Essner R, Hughes TM, Tang PC, Bilchik A, Wanek LA, et al. Surgical resection for metastatic melanoma to the liver: the John Wayne Cancer Institute and Sydney Melanoma Unit experience. Arch Surg. 2001;136:950-5.

34. Karavias DD, Tepetes K, Karatzas T, Felekouras E, Androulakis J, et al. Liver resection for metastatic noncolorectal non-neuroendocrine hepatic neoplasms. Eur J Surg Oncol. 2002;28:135-9.

35. Ramírez CP, Santoyo J, Domínguez ME, García CE, Cobo M, Suárez MA, et al. Carcinoma suprarrenal: supervivencia a 7 años libre de enfermedad tras resección completa del tumor primario y resecciones repetidas de recidivas locorregional y a distancia. Revisión a raíz de un caso con una pobre esperanza de vida inicial. Arch Esp Urol. 2005;58:115-9. 